\title{
HOLOMORPHIC MAPS OF RIEMANN SURFACES AND WEIERSTRASS POINTS
}

\author{
Masaharu Tanabe \\ To the memory of Professor Nobuyuki Suita
}

\begin{abstract}
We investigate holomorphic maps between hyperelliptic Riemann surfaces and prove rigidity theorems given by correspondences of Weierstrass points.
\end{abstract}

\section{Introduction}

In this paper, all of the Riemann surfaces will be compact and of genera greater than 1 if not specified. Concerning automorphisms of Riemann surfaces, the induced permutations of Weierstrass points determine the automorphisms. Namely, if an automorphism $T$ on a Riemann surface $X$ fixes all of the Weierstrass points, then $T=\mathrm{id}$. or $X$ is hyperelliptic and $T$ is the hyperelliptic involution. This fact implies that the number of automorphisms is finite (Schwarz). It is natural to ask what happens when the target Riemann surface is different from the source. Let $X$ and $Y$ be compact Riemann surfaces and let $h: X \rightarrow Y$ be a nonconstant holomorphic map. Martens [Mr] pointed out the following (for Riemann surfaces of positive genera). If $D=\sum m_{i} Q_{i}$ is a positive divisor of degree $n$ and (projective) dimension $r$ on $X$, then $h(D)=$ $\sum m_{i} h\left(Q_{i}\right)$ is a positive divisor of degree $n$ and dimension $\geq r$ on $Y$. If the complete linear system determined by $D$ is without fixed points, then so is that determined by $h(D)$. Hence it follows that when $X$ is hyperelliptic, $Y$ is also hyperelliptic and every Weierstrass point on $X$ maps on a Weierstrass point on $Y$. We denote by $W$ the set of Weierstrass points on $X$. We will show

THEOREM 1. Let $X$ be a hyperelliptic Riemann surface and let $Y$ be a Riemann surface. Let $h_{1}, h_{2}: X \rightarrow Y$ be nonconstant holomorphic maps (then $Y$ is also hyperelliptic). If $\left.h_{1}\right|_{W}=\left.h_{2}\right|_{W}$, then $h_{1}=h_{2}$ or $h_{1}=\tau \circ h_{2}$, where $\tau$ is the hyperelliptic involution.

2000 Mathematics Subject Classification. Primary 14H55; Secondary 14H40.

Partially supported by Grant-in-Aid for Scientific Research (No. 14540155, (C)(2)), Japan Society for the Promotion of Science.

Received March 30, 2004; revised July 23, 2004. 
This assertion gives an upper bound for the number of nonconstant holomorphic maps between hyperelliptic Riemann surfaces depending only on the number of Weierstrass points (or, we can say, on the genus).

We may put another interpretation on Theorem 1 in terms of homology groups. A holomorphic map $h: X \rightarrow Y$ induces a homomorphism

$$
h_{* n}: H_{1}\left(X, \mathbf{Z}_{n}\right) \rightarrow H_{1}\left(Y, \mathbf{Z}_{n}\right)
$$

between the first homology groups with coefficients in the integers $\bmod n$. For automorphisms, denote by $\phi: \operatorname{Aut}(X) \rightarrow \operatorname{Sp}\left(g, \mathbf{Z}_{n}\right)$ the natural homomorphism, where $\operatorname{Sp}\left(g, \mathbf{Z}_{n}\right)$ is the symplectic group of genus $g$ over $\mathbf{Z}_{n}$. Then, it is shown that for $n>2, \phi$ is injective, and for $n=2$, only automorphisms of order 2 is in the kernel of $\phi$ (for the proof see $[\mathrm{F}-\mathrm{K}]$ Chapter 5). For holomorphic maps, if two holomorphic maps $h_{i}: X \rightarrow Y(i=1,2)$ induce the same homomorphism $H_{1}\left(X, \mathbf{Z}_{n}\right) \rightarrow H_{1}\left(Y, \mathbf{Z}_{n}\right)$ for some $n>\sqrt{8(g-1)}$ where $g$ is the genus of $X$, then $h_{1}=h_{2}$ (see [T]). For hyperelliptic case, we may take $n=2$.

THEOREM 2. Let $X$ be a hyperelliptic Riemann surface and let $Y$ be a Riemann surface. Let $h_{1}, h_{2}: X \rightarrow Y$ be nonconstant holomorphic maps (then $Y$ is also hyperelliptic). If $h_{1}$ and $h_{2}$ induce the same homomorphism $H_{1}\left(X, \mathbf{Z}_{2}\right) \rightarrow$ $H_{1}\left(Y, \mathbf{Z}_{2}\right)$, then $h_{1}=h_{2}$ or $h_{1}=\tau \circ h_{2}$.

Further, we will investigate the case where the target surface is not fixed.

For hyperelliptic Riemann surfaces, the case where the covering is normal has been studied (see $[\mathrm{F}],[\mathrm{H}],[\mathrm{K}]$ and $[\mathrm{Mc}]$ ).

\section{Preliminaries}

Let $X$ be a compact Riemann surface of genus $g>1$. Recall that for each point $p \in X$, there are $g$ integers called gaps

$$
1=n_{1}<n_{2}<\cdots<n_{g}<2 g
$$

such that there does not exist a meromorphic function on $X$ holomorphic on $X-\{p\}$ with a pole of order $m$ at $p$ if and only if $m=n_{i}$. Except only a finite number of points, $\left\{n_{1}, \ldots n_{g}\right\}=\{1, \ldots, g\}$ and the excepted points are called Weierstrass points. We denote by $W$ the set of Weierstrass points. Then, the number of Weierstrass points $\# W$ satisfies

$$
2 g+2 \leq \# W \leq g^{3}-g .
$$

A Riemann surface with a meromorphic function of degree 2 is called to be hyperelliptic. $X$ is hyperelliptic if and only if $\# W=2 g+2$. Each Weierstrass point on a hyperelliptic Riemann surface of genus $g$ has the gap sequence $\{1,3, \ldots, 2 g-1\}$.

Let $h: X \rightarrow Y$ denotes a nonconstant holomorphic map between Riemann surfaces. The Martens' assertion in the introduction (or a little modification of his proof in $[\mathrm{Mr}]$ ) gives that if $D$ is the polar divsor of some meromorphic function 
on $X$, then $h(D)$ is the polar divisor of some meromorphic function on $Y$. Thus, we see that if $n$ is a non-gap for $p \in X$, then $n$ is also a non-gap for $h(p) \in Y$. Especially,

Lemma 1 (Martens [Mr]). When $X$ is hyperelliptic, $Y$ is also hyperelliptic and every Weierstrass point of $X$ maps on a Weierstrass point of $Y$. Furthermore, the involution of $X$ followed by $h$ is equivalent to $h$ followed by the involution of $Y$.

The first assertion makes the assumption of Theorem 1 meaningful.

A preimage of a Weierstrass point is not always a Weierstrass point even if $X$ is hyperelliptic. We see this by the following example.

Example 1. let $X$ be a hyperelliptic Riemann surface of genus 3 which has an fixed-point-free automorphism of order 2 . Then the quotient surface $Y$ is of genus 2 and there are 6 Weierstrass points on $Y$. Thus, the number of preimages of them is 12, while the number of Weierstrass points on $X$ is 8 .

To prove Theorem 2, we use theory of Jacobian varieties. By definition, $T:=\mathbf{C}^{g} / \Lambda$ is a complex torus, where $\Lambda$ is a lattice in $\mathbf{C}^{g}$. Choose bases $e_{1}, \ldots, e_{g}$ of $\mathbf{C}^{g}$ and $\chi_{1}, \ldots, \chi_{2 g}$ of the lattice $\Lambda$. Write $\chi_{j}$ in terms of the basis $e_{1}, \ldots, e_{g}: \chi_{j}=\sum_{k=1}^{g} \chi_{k j} e_{k}$. We denote by $M(n, m ; K)$ the set of $n \times m$ matrices with $K$-coefficients. The matrix $\Pi=\left(\chi_{k j}\right) \in M(g, 2 g ; \mathbf{C})$ is called a period matrix for $T$. By an underlying real structure for $T$, we mean the real torus $\mathbf{R}^{2 g} / \mathbf{Z}^{2 g}$ together with a map $\mathbf{R}^{2 g} / \mathbf{Z}^{2 g} \rightarrow T$ induced by a linear map $\mathbf{R}^{2 g} \ni x \mapsto \Pi x \in \mathbf{C}^{g}$, where $\Pi$ is a period matrix. Note that $\Pi: \mathbf{R}^{2 g} / \mathbf{Z}^{2 g} \rightarrow T$ is homeomorphic. We denote by $T_{n}(n \in \mathbf{N})$ the group of $n$-division points of $T$, that is, the kernel of the homomorphism $n_{T}: T \rightarrow T$ defined by $\mathbf{z} \mapsto n \times \mathbf{z}(\mathbf{z} \in T)$. On the real torus, $n$-division points are vectors of the form ${ }^{t}\left(m_{1} / n, \ldots, m_{2 g} / n\right), m_{1}, \ldots, m_{2 g} \in \mathbf{Z}$, and we also denote by $\left(\mathbf{R}^{2 g} / \mathbf{Z}^{2 g}\right)_{n}$ the set of $n$-division points on the underlying real torus.

Let $T$ and $T_{1}$ be complex tori of dimension $g$ and $\gamma$, respectively. Denote by $\Pi$ and $\Pi_{1}$ period matrices for $T$ and $T_{1}$, respectively. It is known that for any homomorphism (i.e., a holomorphic map compatible with the group action) $f: T \rightarrow T_{1}$, there are $A \in M(\gamma, g ; \mathbf{C})$ and $M \in M(2 \gamma, 2 g ; \mathbf{Z})$ such that the following diagram is commutative (cf. [L-B], Chapter 1).

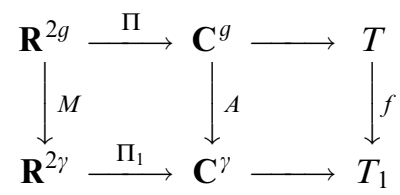

We will call a map $F: \mathbf{R}^{2 g} / \mathbf{Z}^{2 g} \rightarrow \mathbf{R}^{2 \gamma} / \mathbf{Z}^{2 \gamma}$ a linear map if $F$ is induced by a linear map $\tilde{F}: \mathbf{R}^{2 g} \rightarrow \mathbf{R}^{2 \gamma}$ with $\left.\tilde{F}\right|_{\mathbf{Z}^{2 g}} \subset \mathbf{Z}^{2 \gamma}$. By the diagram above, we see that there exists a linear map $F: \mathbf{R}^{2 g} / \mathbf{Z}^{2 g} \rightarrow \mathbf{R}^{2 \gamma} / \mathbf{Z}^{2 \gamma}$ which satisfies $\Pi_{1} \circ F=f \circ \Pi$.

Let $\left\{\chi_{1}, \ldots, \chi_{2 g}\right\}$ be a basis for $H_{1}(X, \mathbf{Z})$ and let $\left\{\omega_{1}, \ldots, \omega_{g}\right\}$ be a basis 
for the space of holomorphic differentials on $X$. By definition, the Jacobian variety $J(X)=\mathbf{C}^{g} / \Lambda$ is the complex torus where $\Lambda$ is the lattice generated by the period matrix with respect to the bases. Let $p_{0} \in X$. Define $\phi_{X}: X \rightarrow J(X)$ by $p \mapsto\left\{\int_{p_{0}}^{p} \omega_{j}\right\}_{j=1}^{g}$. It is known that $\phi_{X}$ is an embedding. Let $h: X \rightarrow Y$ be a holomorphic map of Riemann surfaces. Then, there exists a homomorphism $f: J(X) \rightarrow J(Y)$ which satisfies $f \circ \phi_{X}=\phi_{Y} \circ h$ where $\phi_{X}$ (resp. $\phi_{Y}$ ) is the embedding with the base point $p_{0}$ (resp. $\left.h\left(p_{0}\right)\right)$.

Lemma 2. Let $F_{i}: \mathbf{R}^{2 g} / \mathbf{Z}^{2 g} \rightarrow \mathbf{R}^{2 \gamma} / \mathbf{Z}^{2 \gamma}$ be linear maps $(i=1,2)$. Then, $\left.F_{1}\right|_{\left(\mathbf{R}^{2 g} / \mathbf{Z}^{2 g}\right)_{n}}=\left.F_{2}\right|_{\left(\mathbf{R}^{2 g} / \mathbf{Z}^{2 g}\right)_{n}}$ if and only if $F_{1 * n}=F_{2 * n}$, where $F_{i * n}$ are homomorphisms between homology groups $H_{1}\left(\mathbf{R}^{2 g} / \mathbf{Z}^{2 g}, \mathbf{Z}_{n}\right) \rightarrow H_{1}\left(\mathbf{R}^{2 \gamma} / \mathbf{Z}^{2 \gamma}, \mathbf{Z}_{n}\right)$ induced by $F_{i}$ $(i=1,2)$.

Proof. Suppose $F_{1 * n}=F_{2 * n}$ holds. Let $\mathbf{x} \in\left(\mathbf{R}^{2 g} / \mathbf{Z}^{2 g}\right)_{n}$. Then, it can be written in the form $\mathbf{x}={ }^{t}\left(m_{1} / n, \ldots, m_{2 g} / n\right), m_{1}, \ldots, m_{2 g} \in \mathbf{Z}$. Let $F_{1 *}-F_{2 *}$ : $H_{1}\left(\mathbf{R}^{2 g} / \mathbf{Z}^{2 g}, \mathbf{Z}\right) \rightarrow H_{1}\left(\mathbf{R}^{2 \gamma} / \mathbf{Z}^{2 \gamma}, \mathbf{Z}\right)$ denote the difference of two induced homomorphisms between homology groups with $\mathbf{Z}$-coefficients. Then, the assumption $F_{1 * n}-F_{2 * n}=0$ means that every coefficient of $F_{1 *}-F_{2 *}$ is a multiple of $n$. Recalling that $H_{1}\left(\mathbf{R}^{2 g} / \mathbf{Z}^{2 g}, \mathbf{Z}\right) \simeq \mathbf{Z}^{2 g}$ and that we can identify it with the lattice of the real torus, we see $\left(\tilde{F}_{1}-\tilde{F}_{2}\right)(\mathbf{x}) \in \mathbf{Z}^{2 \gamma}$, equivalently $F_{1}-\left.F_{2}\right|_{\left(\mathbf{R}^{2 g} / \mathbf{Z}^{2 g}\right)_{n}}=0$. Conversely, if $\left.F_{1}\right|_{\left(\mathbf{R}^{2 g} / \mathbf{Z}^{2 g}\right)_{n}}=\left.F_{2}\right|_{\left(\mathbf{R}^{2 g} / \mathbf{Z}^{2 g}\right)_{n}}$ holds, then denoting by $\mathbf{e}_{j}$ the vector whose $j$-th entry is 1 and others are $0, \tilde{F}_{1}-\tilde{F}_{2}$ maps $\mathbf{e}_{j} / n$ to a integral vector for every $j=1,2, \ldots, 2 g$. It implies that every coefficient of $F_{1 *}-F_{2 *}$ is a multiple of $n$ and we see $F_{1 * n}=F_{2 * n}$.

\section{Rigidity theorems}

Proof of Theorem 1. Let $\pi$ be a meromorphic function on $Y$ of degree 2 . Consider 2 meromorphic functions $\pi \circ h_{1}$ and $\pi \circ h_{2}$. Put $F=\pi \circ h_{1}-\pi \circ h_{2}$. Suppose $F \not \equiv 0$. By the Riemann-Hurwitz relation, we see that the degree of $\pi \circ h_{1}$ and $\pi \circ h_{2}$ is $\leq 2(g-1) /(\gamma-1)$. Thus, the degree of the polar divisor of $F$ is $\leq 4(g-1) /(\gamma-1)$. On the other hand, the zero divisor of $F$ is $\geq 4(g+1)$ because each of the Weierstrass points is one of zero points of $F$ and the ramification index of it is at least 2 for a Weierstrass point on $X$ maps on a Weierstrass point on $Y$ and the Weierstrass points on $Y$ is the ramification points of $\pi$. But $4(g+1)>4(g-1) /(\gamma-1)$, contradiction. Thus, we must have $F \equiv 0$, equivalently $\pi \circ h_{1}=\pi \circ h_{2}$. Suppose $h_{1} \neq h_{2}$. Then, for a small neighborhood $U_{p}$ of an arbitrary point $p \in X$, we have $\left.h_{1}\right|_{U_{p}}=\left.\tau \circ h_{2}\right|_{U_{p}}$ and it implies $h_{1}=\tau \circ h_{2}$ by the theorem of identity.

Proof of Theorem 2. Let $\left\{\chi_{1}, \ldots, \chi_{2 g}\right\}$ be a basis for $H_{1}(X, \mathbf{Z})$ and let $\left\{\omega_{1}, \ldots, \omega_{g}\right\}$ be a basis for the space of holomorphic differentials on $X$. Let $J(X)=\mathbf{C}^{g} / \Lambda$ be the Jacobian variety of $X$ where $\Lambda$ is the lattice generated by the period matrix with respect to the bases. Let $p_{0} \in X$. Define $\phi: X \rightarrow J(X)$ 
by $p \mapsto\left\{\int_{p_{0}}^{p} \omega_{j}\right\}_{j=1}^{g}$. Then, if $p_{0}$ is a Weierstrass point, any Weierstrass point $p \in W$ satisfies $\phi(p) \in J(X)_{2}$ and $\{\phi(p)\}_{p \in W}$ generate $J(X)_{2}$ (cf. [F-K] Chapter 7). We denote by $f_{i}: J(X) \rightarrow J(Y)$ the homomorphisms induced by $h_{i}$ and by $F_{i}$ the homomorphism of underlying real tori induced by $h_{i}(i=1,2)$. Then each induced homomorphism between homology groups of underlying real tori (which is the same as the homomorphism between the homology groups of Jacobians) is the same as that of Riemann surfaces. Thus, by the assumption and Lemma 2, $\left.F_{1}\right|_{\left(\mathbf{R}^{2 g} / \mathbf{Z}^{2 g}\right)_{2}}=\left.F_{2}\right|_{\left(\mathbf{R}^{2 g} / \mathbf{Z}^{2 g}\right)_{2}}$. Recalling the diagram in Preliminaries, we see $\left.f_{1}\right|_{J(X)_{2}}=\left.f_{2}\right|_{J(X)_{2}}$. It implies $\left.h_{1}\right|_{W}=\left.h_{2}\right|_{W}$, and by Theorem 1, we get the conclusion.

Now, we turn to the case where the target is not fixed. Let $X$ be a hyperelliptic Riemann surface of genus $g$ and let $Y_{1}$ and $Y_{2}$ be Riemann surfaces of the same genus $\gamma$. Let $h_{i}: X \rightarrow Y_{i}(i=1,2)$ be nonconstant holomorphic maps. In this situation, the condition $\left.h_{1}\right|_{W}=\left.h_{2}\right|_{W}$ is meaningless. Thus, we consider the following combinatorial condition. if

Ordering Weierstrass points $p_{j}^{i}(j=1,2, \ldots 2 \gamma+2)$ on $Y_{i}(i=1,2)$ properly,

$$
W \cap h_{1}^{-1}\left(p_{j}^{1}\right)=W \cap h_{2}^{-1}\left(p_{j}^{2}\right)
$$

holds set-theoretically for every $j=1,2, \ldots 2 \gamma+2$, then we will say that $h_{1}$ and $h_{2}$ satisfy the $W$-condition.

Proposition. Let $h_{i}: X \rightarrow Y_{i}(i=1,2)$ be holomorphic maps, where $Y_{1}$ and $Y_{2}$ are of the same genus $\gamma>4$. If $h_{1}$ and $h_{2}$ satisfy the $W$-condition, then there exist double-covers $\pi_{i}: Y_{i} \rightarrow \hat{\mathbf{C}}(i=1,2)$ which satisfies $\pi_{1} \circ h_{1}=\pi_{2} \circ h_{2}$.

Proof. Without loss of generality, we may assume that the number of elements of sets satisfy $\#\left\{W \cap h_{1}^{-1}\left(p_{1}^{1}\right)\right\} \geq \#\left\{W \cap h_{1}^{-1}\left(p_{2}^{1}\right)\right\} \geq \cdots \geq$ $\#\left\{W \cap h_{1}^{-1}\left(p_{2 \gamma+2}^{1}\right)\right\}$ and double-covers satisfy $\pi_{i}\left(p_{1}^{i}\right)=1, \pi_{i}\left(p_{2}^{i}\right)=2$ and $\pi_{i}\left(p_{3}^{i}\right)=$ $3(i=1,2)$. Put $F=\pi_{1} \circ h_{1}-\pi_{2} \circ h_{2}$. Then, $W \cap h_{1}^{-1}\left(p_{j}^{1}\right)(j=1,2,3)$ are zeros of $F$ and so the degree of zero divisor of $F$ is $\geq 2 \times 3 \times(g+1) /(\gamma+1)$. On the other hand, if $F \neq 0$, the degree of polar divisor of $F$ is $\leq 2 \times 2 \times$ $(g-1) /(\gamma-1)$ where $(g-1) /(\gamma-1)$ comes from the maximum degree for $h_{i}(i=1,2)$. Assumption $\gamma>4$ leads us to the conclusion that $F \equiv 0$.

If there is a conformal map $S: Y_{1} \rightarrow Y_{2}$ with $S \circ h_{1}=h_{2}$, then we will write $h_{1} \simeq h_{2}$. Even if $\pi_{1} \circ h_{1}=\pi_{2} \circ h_{2}$, it does not always mean $h_{1} \simeq h_{2}$. But for unramified cases, we have

THEOREM 3. Let $X$ be a hyperelliptic Riemann surface. Let $h_{i}: X \rightarrow Y_{i}$ $(i=1,2)$ be nonconstant holomorphic maps where $Y_{i}(i=1,2)$ are of the same genus $\gamma>4$. If $h_{i}(i=1,2)$ are unramified and satisfy the $W$-condition, then $h_{1} \simeq h_{2}$. 
Proof. By Proposition, we have $\pi_{1} \circ h_{1}=\pi_{2} \circ h_{2}$. All of the branch points on $\hat{\mathbf{C}}$ are image of Weierstrass points on $Y_{i}$ since $h_{i}$ are unramified $(i=1,2)$. Thus, $Y_{1}$ and $Y_{2}$ are conformally equivalent since they are expressed by the same algebraic function as a double-cover over $\hat{\mathbf{C}}$. Applying Theorem 1, we see $h_{1} \simeq h_{2}$.

In this case, we also have a theorem in terms of homology groups. Let $G, G_{1}$ and $G_{2}$ be groups and let $H_{1}: G \rightarrow G_{1}$ and $H_{2}: G \rightarrow G_{2}$ be homomorphisms. If there exists a isomorphism $\psi: G_{1} \rightarrow G_{2}$ such that $\psi \circ H_{1}=H_{2}$, we will say $H_{1}$ and $H_{2}$ are isomorphic.

THEOREM 4. Let $X$ be a hyperelliptic Riemann surface. Let $h_{i}: X \rightarrow Y_{i}$ $(i=1,2)$ be nonconstant holomorphic maps where $Y_{i}(i=1,2)$ are of the same genus $\gamma>4$. If $h_{i}(i=1,2)$ are unramified and induced homomorphisms $h_{i * 2}: H_{1}\left(X, \mathbf{Z}_{2}\right) \rightarrow H_{1}\left(Y_{i}, \mathbf{Z}_{2}\right)(i=1,2)$ are isomorphic, then $h_{1} \simeq h_{2}$.

Proof. We use underlying real structures. On underlying real tori, choosing homology bases properly, linear maps $F_{i}: \mathbf{R}^{2 g} / \mathbf{Z}^{2 g} \rightarrow \mathbf{R}^{2 \gamma} / \mathbf{Z}^{2 \gamma}$ induced by $h_{i}(i=1,2)$ satisfy $F_{1 * 2}=F_{2 * 2}$. By Lemma 2 , it is equivalent to $\left.F_{1}\right|_{\left(\mathbf{R}^{2 g} / \mathbf{Z}^{2 g}\right)_{2}}$ $=\left.F_{2}\right|_{\left(\mathbf{R}^{2 g} / \mathbf{Z}^{2 g}\right)_{2}}$. Recall the diagram in Preliminaries and that any Weierstrass point $p \in W$ satisfies $\phi(p) \in J(X)_{2}$ if we choose a Weierstrass point as the base point of the embedding. Then we see that $h_{1}$ and $h_{2}$ satisfy the W-condition. Applying Theorem 3, we get the conclusion.

When $h_{i}(i=1,2)$ are ramified, Theorem 3, 4 do not hold. We see this by the following example.

Example 2. We picture two copies of the sphere $\hat{\mathbf{C}}$. We label these two copies sheet 1 and sheet 2 . Each sheet, we cut along segments $[-1,1],[2,3]$, $[-2,-3],[4,5],[-4,-5],[6,7],[-6,-7], \ldots,[10,11],[-10,-11]$. Each cut is considered to have two banks; a +bank and a -bank. We construct a Riemann surface $R$ by joining every + bank on sheet 1 to a -bank of the corresponding cut on sheet 2 , and then joining the corresponding + bank on sheet 2 to the -bank of the corresponding cut on sheet 1 . Then $R$ is a hyperelliptic Riemann surface of genus 10 and $z$ is a double-cover of $\hat{\mathbf{C}}$. Rotation of angle $\pi$ of center 0 on $\hat{\mathbf{C}}$, that is, $z \mapsto-z$, can be extended to $R$ and we denote the extended map by $T$. We put $Y_{1}:=R /\langle T\rangle$ and $Y_{2}:=R /\langle\tau \circ T\rangle$, where $\tau$ is the involution. Then, $Y_{1}$ and $Y_{2}$ are both of genus 5 . Let $h_{i}: R \rightarrow Y_{i}$ be the projections for $i=1,2$. Then, $h_{1}$ and $h_{2}$ satisfy the W-condition. $Y_{1}$ is the double-cover of $\hat{\mathbf{C}}$ branched over $0,1,2, \ldots, 11$. On the other hand, $Y_{2}$ is the double-cover of $\hat{\mathbf{C}}$ branched over $1,2, \ldots, 11, \infty$. We see that $Y_{1}$ and $Y_{2}$ are not conformally equivalent (e.g., comparing cross ratios of branch points).

With slight and obvious modification, we can construct such examples for higher (and also lower) genera. 


\section{REFERENCES}

[F-K] H. M. FARKas And I. Kra, Riemann surfaces, Springer-Verlag, New York, Heidelberg and Berlin, 1980.

[F] H. M. FARKAS, Unramified double coverings of hyperelliptic surfaces, J. Analyse Math. 30 (1976), 150-155.

[H] R. HoriuchI, Normal coverings of hyperelliptic Riemann surfaces, J. Math. Kyoto Univ. 19 (1979), 497-523.

[K] T. Kato, On the hyperellipticity of coverings of Riemann surfaces, (unpublished).

[L-B] H. Lange And C. Birkenhake, Complex abelian varieties, Springer-Verlag, New York, Heidelberg and Berlin, 1992.

[Mc] C. Maclachlan, Smooth coverings of hyperelliptic surfaces, Qurt. J. Math. (2) 22 (1971), 117-123.

[Mr] H. Martens, A remark on Abel's theorem and the mapping of linear series, Comment. Math. Helvetica 52 (1977), 557-559.

[T] M. Tanabe, On rigidity of holomorphic maps of Riemann surfaces, Osaka J. Math. 33 (1996), 485-496.

Department of Mathematics

Tokyo Institute of Technology

Ohokayama, Meguro, Tokyo, 152-8551

JAPAN

E-mail address: tanabe@math.titech.ac.jp 